\title{
The effect of the plastic instability on the behavior of an amorphous polymer
}

\author{
Ali B.M. ${ }^{1,2 *}$, Bouiadjera B.B. ${ }^{1}$, Chikh E.B.O. ${ }^{2}$, Elmeguenni M. ${ }^{1}$ \\ ${ }^{1}$ Faculty Technology, Mechanical Engineering Department, University of Sidi Bel Abbes, Algeria \\ ${ }^{2}$ LSTE laboratories, Faculty of Science and Technology, University of Mascara, Algeria
}

Email: boudjellel.moulai@univ-sba.dz

\begin{abstract}
In manufacturing operations, numerous parts and components are formed into different shapes by applying external forces to the workpiece, typically by means of various tools and dies. Common examples of such operations are forging, extruding, drawing and rolling. Forming operations may be carried out at room temperature or at elevated temperatures, and at a low or a high rate of deformation. These operations are also used in forming and shaping non-metallic materials such as plastics and ceramics. However, the processes of working of polymers in a solid state are affected by phenomena of plastic instabilities. These phenomena are interpreters on the basis of intrinsic law of behavior of the material which express the influence of the deformation and the rate of deformation on the plastic yield stress. This law directly reflects the structural modifications undergone by material under the conditions of the test. In addition, she gives the necessary information to the modeling of instabilities using techniques of calculation such as the finite element method.
\end{abstract}

Keywords: Amorphous Polymer, Damage, Mechanical Behavior, Modeling.

\section{INTRODUCTION}

The use of cylindrical forms structures in polymers has become increasingly common in many areas of activity such as mechanical water lines, gas pipelines, and even in biomechanics such as thin tubes and the blood vessels. However, these structures are often subjected to severe workings (internal \& external constraints). Under the effect of loads and / or internal pressure or temperature changes, these structures can undergo significant deformation where damage can easily occur with the risk to a fatal destruction. For this, the characterization and determination of deformation mechanisms and damage in a more advanced level of these materials body play a very important role to prevent or predict their ruins. During recent years. The common point of all major industrial polymers is to be made up of long macromolecular chains. Each macromolecule is made of several tens to several thousands of identical monomers whose skeleton is most often based investigation of the mechanical behavior of polymers in large deformation has been the subject of many studies and is still the subject of numerous publications.

Polymers such as polyvinyl chloride (PVC), is required increasingly in the transportation sector energy and building. Meanwhile, a major effort is made by the scientific

community to study this type of polymer. So far, the establishment of the mechanical behavior of PVC with the loading conditions posed major plastic instability problems.
In this context, our work consists in studying of the Mechanical behavior of PVC in great deformations and its evolution with respect to the stress triaxiality.

\section{SPECIFIC CHARACTERISTICS OF THE STRUCTURE AND PROPERTIES OF SOLID POLYMERS}

\subsection{Recalls on the structure of polymers}

The common point of all major industrial polymers is to be made up of long macromolecular chains. Each macromolecule is made of several tens to several thousands of identical monomers whose skeleton is most often based on carbon atoms. The atomic bonds along a chain are of covalent type (Figure 1) their energy is very high $(\sim 100 \mathrm{kcal}$ per mole), so that in first approximation we can consider that, they cannot break under the effect of an applied mechanical stress. Despite their strength, the polymer chains can be some flexibility if the temperature is sufficient [03], The secondary interactions are generally type Van-DerWaals and their energy are only about ( $1 \mathrm{kcal}$ per mole) is much lower than that of covalent bonds in the chain. It is this duality of bonds in the polymer structure, which is at the origin of their special mechanical properties.

From the standpoint of general physical properties, we usually recognize three types of solid polymers: elastomers, thermoplastic polymers, and thermosetting polymers. Elastomers are rubbers or rubberlike elastic materials. Thermoplastic polymers are hard at room temperature, but on heating become soft and more or less fluid and can be molded. 
Thermosetting polymers can be molded at room temperature or above, but when heated more strongly become hard and infusible. These categories overlap considerably but are nonetheless helpful in defining general areas of utility and types of structures.

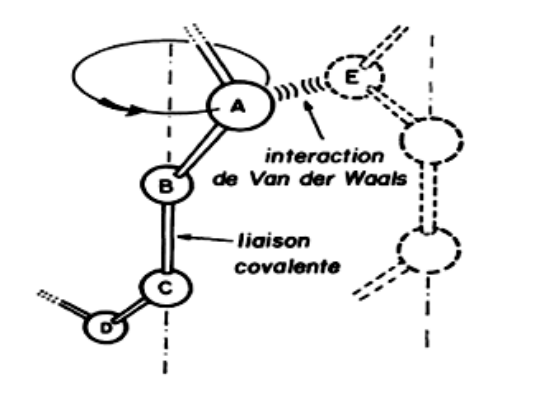

Figure 1. Schematic diagram of the interactions types of a macromolecular chain [03].

The structural characteristics that are most important to determining the properties of polymers are: (1) the degree of rigidity of the polymer molecules, (2) the electrostatic and van der Waals attractive forces between the chains, (3) the degree to which the chains tend to form crystalline domains, and (4) the degree of cross-linking between the chains.

All the examples considered in this article will thus relate to thermoplastic polymers with chemically independent macromolecules.

\subsection{Amorphous and crystalline polymers}

An amorphous polymer is one with no crystallites. If the attractive forces between the chains are weak and if the motions of the chain are not in some way severely restricted as by cross-linking or large rotational barriers, such a polymer would be expected to have low tensile strength and when stressed to undergo plastic flow in which the chains slip by one another.

Polymers whose macromolecular chains are more regular adopt a semi crystalline structure. (Schultz 1974) To minimize the energy conformation, the chains fold in on themselves to form regular segments, which are stacked in a crystal lattice of higher density than the amorphous polymer.

The effect of temperature on the physical properties of polymers is very important to their practical uses. At low temperatures, polymers become hard and glasslike because the motions of the segments of the polymer chains with relation to each other are slow.

The approximate temperature below which glass like behavior is apparent is called the glass temperature and is symbolized by $T g$. When a polymer containing crystallites is heated, the crystallites ultimately melt, and this temperature is usually called the melting temperature and is symbolized as Tm. Usually, the molding temperature will be above Tm, and the mechanical strength of the polymer will diminish rapidly as the temperature approaches Tm.

Figure 3 shows the typical evolution of the elastic modulus of an amorphous polymer with temperature [01].
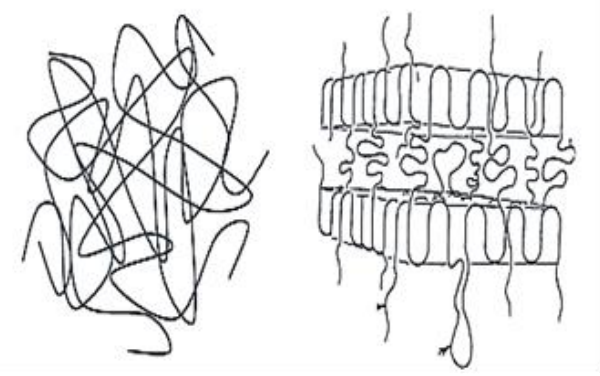

Figure 2. Amorphous and semi-crystalline structure of the polymers

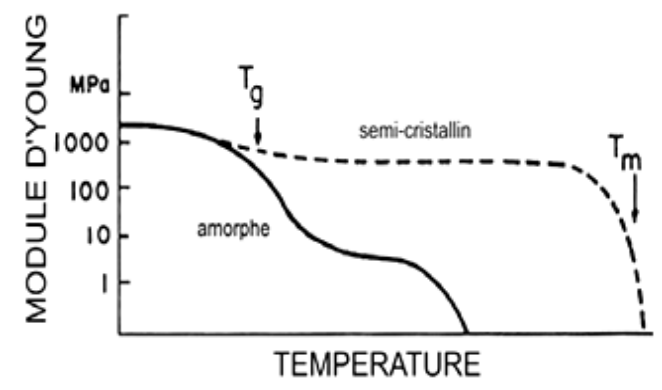

Figure 3. Typical evolution of the elastic modulus of the amorphous and semi-crystalline polymers with temperature

\section{MECHANICAL TESTS}

Polymer components, like other materials, may fail to perform their intended functions in specific applications as a result of:

- Excessive elastic deformation

- Yielding or excessive plastic deformation

- Fracture

Polymers show excessive elastic deformation, particularly in structural, load-bearing applications, due to inadequate rigidity or stiffness. For such failure, the controlling material mechanical property is the elastic modulus. As we shall see in subsequent discussions, the elastic moduli of some polymers are subject to some measure of control through appropriate structural modification.

Failure of polymers in certain applications to carry design loads or occasional accidental overloads may be due to excessive plastic deformation resulting from the inadequate strength properties of the polymer. For the quantification of such failures, the mechanical property of primary interest is the yield strength and the corresponding strain. The ultimate strength, along with the associated strain, also provides useful information.

\subsection{Stress-strain experiments}

The tension test is the most common method for determining the mechanical properties of materials, such as strength, ductility, toughness, elastic modulus, and strain hardening capability. The tension test first requires the preparation of a test specimen, as shown in (Figure 4a), although most tension-test specimens are solid and round, they can also be flat or tubular. The specimen is prepared generally according to ASTM specifications; various other specifications are also available from corresponding organizations around the world. Typically, the specimen has 
an original gage length 10 , and a cross-sectional area D0. It is mounted in the jaws of a tension-testing machine (Figure $4 \mathrm{~b}$ ) equipped with various accessories and controls so that the specimen can be tested at different temperatures and rates of deformation.

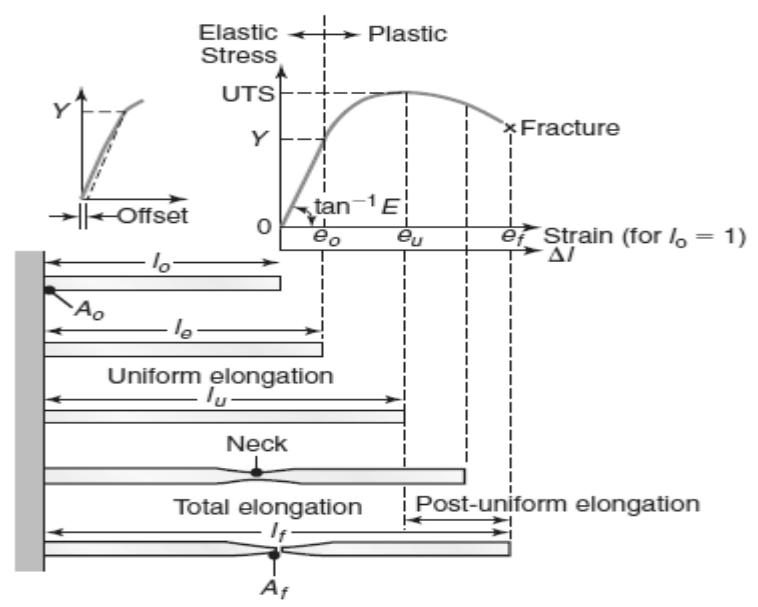

Figure 4. A typical tensile-testing machine (Hosford 2005).

\subsection{Stress-strain behaviors of polymers}

In stress-strain experiments, a polymer sample is pulled (deformed) at a constant elongation rate, and stress is measured as a function of time [02]. Generally,

the polymer specimen, which may be rectangular or circular in cross-section, is molded or cut in the form of a dog bone. It is clamped at both ends and pulled at one of the clamped ends (usually downward) at constant elongation. The shape of the test specimen is designed to encourage failure at the thinner middle portion. The central section between clamps is called the initial gauge length, L0. The load or stress is measured at the fixed end by means of a load transducer as a function of the elongation, which is measured by means of mechanical, optical, or electronic strain gauges. The experimental data are generally stated as engineering (nominal) stress $(\sigma)$ vs. engineering (nominal) strain $(\varepsilon)$. The engineering stress is defined as:

$\sigma=\frac{F}{A_{0}}$

where $\mathrm{F}=$ the applied load.

A $0=$ the original cross-sectional area over the specimen.

This stress, defined with reference to the undeformed configuration, is called engineering stress. If the reduction of the cross-sectional area is large, the engineering stress definition becomes inappropriate. For instance, it fails to predict strain hardening simply because of the use of a constant initial area, A0. A more realistic stress definition should use the instantaneous cross sectional area A:

$\sigma=\frac{F}{A}$

The definition of the stress $\mathrm{s}$ is based on the instantaneous material configuration. It is called the true stress.

Strain describes quantitatively the degree of deformation of a body. It is measured most commonly with extensometers and strain gauges. For uniaxial deformation, strain can be expressed as:
$\varepsilon=\frac{\mathrm{L}-\mathrm{L}_{0}}{\mathrm{~L}_{0}}=\frac{\Delta L}{L_{0}}$

where: L0 original length of the undeformed specimen. L: instantaneous gauge length. $\Delta \mathrm{L}$ : elongation or change in the gauge length.

This strain is the engineering strain, or conventional strain. Based on this definition, if a sample were stretched such that $\mathrm{L}_{\mathrm{f}}=2 \mathrm{~L}_{0}$, the tensile engineering strain would be $100 \%$. On the other hand, if a sample were compressed to the limit such that $\mathrm{L}_{\mathrm{f}}=0$, the compressive engineering strain would again be $100 \%$. These extreme examples show that for large strain the definition of Eq (3) is not meaningful [07].

For purely elastic deformation, stresses are uniquely defined by the final configuration of a material, regardless of how this final state is reached. Because of the presence of irreversible elements in the deformation, a plastic analysis has to follow the path along which the final configuration is reached. To achieve this, the total deformation is generally divided into small increments. Considering the uniaxial case, let $\mathrm{dL}$ be the incremental change in gauge length and $\mathrm{L}$ the gauge length at the beginning of that increment. Then, the corresponding strain increment becomes:

$\mathrm{d} \varepsilon=\frac{\mathrm{dL}}{\mathrm{L}}$

In addition, the total strain for a change of the gauge length from $\mathrm{L}_{0}$ to $\mathrm{L}_{\mathrm{f}}$

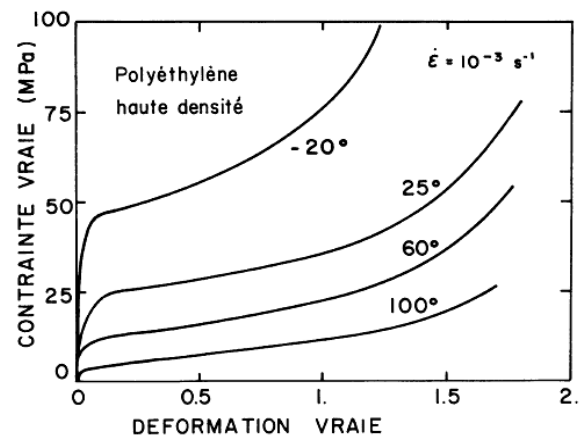

Figure 6. Plastic behavior (true stress vs. true strain) of Highdensity Polyethylene at various temperatures [03]

$\varepsilon=\int_{0}^{\epsilon} \mathrm{d} \varepsilon=\int_{\mathrm{L}_{0}}^{\mathrm{L}_{\mathrm{f}}} \frac{\mathrm{dL}}{\mathrm{L}}=\ln \frac{\mathrm{L}_{\mathrm{f}}}{\mathrm{L}_{0}}$

The strain defined by $\mathrm{Eq}(5)$ is the true strain or natural strain. It is a more suitable definition of strain and is particularly useful for large strain analyses.

\subsection{Typical behavior of solid polymers}

$G^{\prime}$ Sell studied the influence of the temperature on the plastic behavior of polymer [03], In the case of PMMA, (Figure 5) shows that at room temperature, the polymer glass breaks prematurely because the development of micro-cracks ahead of the generalized plasticity. From $70 \mathrm{C}^{\circ}$, it is the plastic flow, which is dominating.

A hook of traction occurs at the end of the viscoelastic stage while microbandes of shearingin the center of the sample multiply.

The low slope below the hook corresponds to a low initial hardening, while the fast recovery of the curve at the end of 
the test indicate the increasingly marked orientation of the chains.

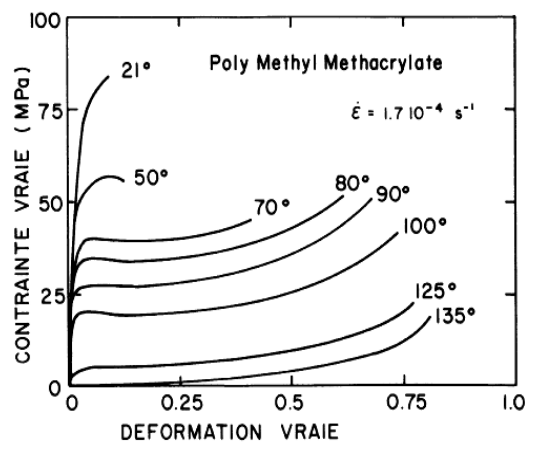

Figure 5. Plastic behavior (true stress vs. true strain) of amorphous PMMA at various temperatures[03].

Above $\mathrm{Tg}$, towards $120 \mathrm{C}^{\circ}$, the threshold of plasticity completely disappears (no visible elastic limit) and the sample returns quickly at the origin if it is released in the course of test: the PMMA then became an elastomer hyper elastic.

The High-Density Polyethylene-HDPE-of (figure 6) is for its part a typical example of semi crystalline polymer. Its intrinsic curve also varies with the temperature but its form varies with the temperature but its form remains practically unchanged since the cryogenic temperatures (where a ductilBrittle transition appears) until the melting point, towards 130 $\mathrm{C}^{\circ}$, where it passes brutally in a viscous state. It is note that the yield stress does not present any more a hook but a progressive round transition. Very strong deformations are applicable, until rates of lengthening of the order of $750 \%$ ( $\varepsilon$ $=2)$.

\section{EXPERIMENTAL PROCEDURE AND RESULTS}

\subsection{Specimen preparation}

Poly (vinyl chloride) (PVC)) was chosen as the model amorphous polymer in the present study, in part because it has already been the subject of previous investigations of the necking process [09]. The material selected was produced by the STPM CHIALI Company certified to ISO 9001 - 2000, and is available commercially as tubes. Its physical and macromolecular characteristics are summarized in Table 1.

Table 1. Technical and physical specifications of PVC.

\begin{tabular}{|l|l|}
\hline Characteristic & \multicolumn{1}{|c|}{ Values } \\
\hline Relative density & $1,3-1,45$ \\
Réfractive index & 1,54 \\
Deflection Temperature ' $1.82 \mathrm{MPa}^{\prime}\left({ }^{\circ} \mathrm{C}\right)$ & 62 \\
Softening point $\left({ }^{\circ} \mathrm{C}\right)$ & 70 \\
Maximum working temperature $\left({ }^{\circ} \mathrm{C}\right)$ & 60 \\
thermal expansion coefficient $(\mathrm{Cm} / \mathrm{cm}$ & à $20^{\circ} \mathrm{C}: 6,7 \times 10^{-5}$ \\
${ }^{\circ} \mathrm{C}$ K-1) & \\
Specific heat capacity & 0.26 \\
Thermal conductivity & $4,5 \times 10^{-4}$ \\
Index of fluidity & $0,2-1,4 \mathrm{~g} / 10(\mathrm{~min})$ \\
average molar mass $\mathrm{Mw}$ & $310000(\mathrm{~g} / \mathrm{mol})$ \\
Melting temperature Tm & $130\left({ }^{\circ} \mathrm{C}\right)$ \\
\hline
\end{tabular}

\subsection{Setting a constitutive law}

We mean by a constitutive law, a mathematical relationship that describes the dependence of stress to strain and tensile strain rate at a given temperature, this law constitutes the equation of material's state insofar as the parameter of deformation $\varepsilon$ characterizes in a suitable way the structural state of material.

This property is checked only at first approximation because for a value of fixed deformation, the structure of a polymer can evolve by the set of phenomena of relieving of the chains, However, it has been shown by G'Sell and Jonas [03] for a stretching made under normal conditions, this restriction arises only during very limited transitional periods, for example at the beginning of plastic stage (pulling hook) or after a period when the plastic strain rate is suddenly changed (a speed jump). In general, we can consider that the deformation is a good indicator of the structural state of a polymer material, because it is linked to the texture orientation adopted by the chains during plastic deformation This is confirmed by the fact that if a specimen is discharged after stretching, deformation and structure tend to return to their original state. Under these assumptions, it has been shown by G'Sell that of most polymers behavior law can be written in a single simplified form:

$\sigma\left(\varepsilon, \varepsilon^{*}\right)=K \cdot f(\varepsilon) \cdot g(\varepsilon)$

where $K$ is a scale factor while $f(\varepsilon)$ and $g(\varepsilon)$ showing the influence of the deformation and strain rate respectively. More specifically, the function $f(\varepsilon)$ can be written as a first approximation:

$f(\varepsilon)=V(\varepsilon) \cdot C(\varepsilon) \cdot H(\varepsilon)$

where, $V(\varepsilon)$ describes the viscoelastic behavior as follows:

$V(\varepsilon)=1-\exp \left(-W \varepsilon^{D}\right)$

$C(\varepsilon)$ takes into account a possible stretching hook:

$C(\varepsilon)=1+a \cdot \exp (-b \varepsilon)$

$H(\varepsilon)$ describes the gradual hardening to large deformations:

$H(\varepsilon)=\exp \left(h \varepsilon^{n}\right)$

where the exponent $n$ has a value close to 2 in most cases.

The rate sensitivity, expressed by the function $g\left(\varepsilon^{*}\right)$ can be put in the form of a power law:

$g(\varepsilon)=\varepsilon^{m}$

The dependence of the stress to the variables $\varepsilon$ and $\varepsilon$ are often expressed as partial derivatives of the first order(Jonas, Holt et al. 1976).

$\left\{\begin{array}{l}\gamma=(\partial \ln \sigma / \partial \varepsilon)_{\varepsilon} \\ m=(\partial \ln \sigma / \partial \varepsilon)_{\varepsilon}\end{array}\right.$

$y$ relative hardening coefficient and $m$ coefficient of sensitivity to the strain rate. 
It can be seen that $\mathrm{Eq}(6)$ separates variable $\varepsilon$ and $\varepsilon$ under multiplicative form, so that $\gamma$ depend only on $\varepsilon$ and $m$ would depend only of $\varepsilon$, this property is fairly well verified in practice as a first approximation.

However, for some applications, it is necessary to take account of coefficients of the second order:

$\left\{\begin{array}{c}A=(\partial \gamma / \partial \ln \varepsilon)_{\varepsilon} \\ B=(\partial m / \partial \varepsilon)_{\varepsilon}\end{array}\right.$

These cross influences are expressed primarily by small variations in $V(\varepsilon)$ and $C(\varepsilon)$ with $\varepsilon$ and exponent $m$ with $\varepsilon$.

In order to reproduce the evolution of the behavior of our $\mathrm{PVC}$, it is interesting to compare the two laws proposed by G'Sell behavior. The first law is rather simple and is written as follows

$$
\sigma=K \cdot(1-\exp (-w \cdot \varepsilon)) \cdot \exp \left(h \cdot \varepsilon^{2}\right) \cdot \varepsilon^{m}
$$

The second law is more complex than the first, and is often written as following form:

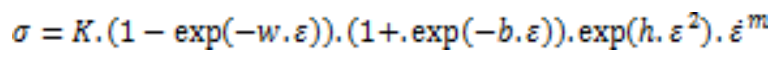

In the previous section, stress and strain are defined for the simple uniaxial state of stress. This simplified the introduction of the basic concept of stress and strain without using tensors. More importantly, the uniaxial state of stress is one of the few simple cases in which the stress-strain relation may be verified experimentally.

A general theory of plasticity must deal with states of stress comprising six stress components $\sigma_{x,} \sigma_{y^{v}} \sigma_{z,} \tau_{x y y}, \tau_{y z} \tau_{x z}$ and six strain components $\varepsilon_{x^{x}} \varepsilon_{y^{x}} \varepsilon_{z}, \gamma_{x y s} \gamma_{y z}, \gamma_{x z}$. Note that here the conventional notations for stresses and strains are used. The uniaxial state of stress is a special case where there is only one nonzero normal stress

$\left\{\begin{array}{c}\sigma_{x}=\sigma \\ \sigma_{y}=\sigma_{z}=\tau_{x y}=\tau_{y z}=\tau_{x z}=0\end{array}\right.$

If the material is isotropic, the strains in the uniaxial state are:

$\left\{\begin{array}{c}\varepsilon_{x z}=\varepsilon \\ \varepsilon_{y}=\varepsilon_{z}=-\varepsilon / 2 \\ \gamma_{x y}=\gamma_{y z}=\gamma_{x z}=0\end{array}\right.$

Generally, only the stress-strain relation in the uniaxial state is known. Therefore, one needs to relate stresses and strains in a general state to uniaxial observations. For this purpose, it is assumed that for any given stress state, there exists an equivalent uniaxial stress state. The equivalent uniaxial stress, or the von Mises, stress is defined as:

$$
\sigma_{e q}=\sqrt{\frac{1}{2}}\left[\left(\sigma_{x x}-\sigma_{y y}\right)^{2}+\left(\sigma_{y y}-\sigma_{z z}\right)^{2}+\left(\sigma_{z z}-\sigma_{x x}\right)^{2}\right]
$$

\subsection{The effective stress and the Bridgman triaxiality factor}

The analysis described in Section 4.2 above is rigorously valid only when the state of stress is uniaxial, that is when no radial or circumferential stresses are developed in the stretched bar. Assumption is reasonably valid as long as the tensile deformation is homogeneous and the profile of the specimen remains fairly uniform. Conversely, if the stress tensor deviates from a uniaxial one, so that finite radial and tangential stresses ( $\sigma y$ and $\sigma z$, respectively) are induced in addition to the axial stress Ox, the plastic behavior of the specimen must be described instead in terms of the local effective stress $\sigma_{\text {eff }}$ and effective strain $\varepsilon_{\text {eff }}$ [04].

According to this concept, $\sigma_{\text {eff }}$ is numerically equal to that uniaxial stress which would produce the same effective strain rate as the one generated by the complex stress field. The rate of plastic deformation is increased by the triaxiality effect if the transverse stresses are compressive and conversely decreased if the hydrostatic term in tensile.

\subsection{Correction method}

The average axial or nominal stress, $(\sigma)$, at the smallest cross section is: $\sigma=F / A$

Because radial and tangential stresses, $\sigma y$ and $\sigma z$, are not zero the nominal stress is not the equivalent uniaxial stress. Using the stress equilibrium equations at the minimum section, Bridgman3 gives the stress distribution at the smallest cross section as:

$$
\begin{aligned}
& \sigma_{x x}=\sigma_{y y}=\sigma \log \frac{a^{2}+2 a R-x^{2}}{2 a R} \\
& \sigma_{z z}=\sigma\left(1+\log \frac{a^{2}+2 a R-x^{2}}{2 a R}\right)
\end{aligned}
$$

where $x$ is the radial distance in the minimum section of the neck, and $R$ is the radius of curvature of the neck profile.

In practice, $a=D / 2$ and the radius of curvature $R$ are automatically measured by a video device controlled by computer. To assure a constant strain rate $\dot{\varepsilon}$, it is necessary to slave the crossbeam of the tensile testing machine so that the diameter decreases according to the law $\mathrm{D}=\mathrm{Do} \exp \left(-\frac{\vec{\varepsilon}}{\mathrm{\varepsilon}} \mathrm{t} / 2\right)$ [03].

Because the shear stresses disappear at the smallest part of the cross section, the equivalent uniaxial stress calculated from Eq (18) becomes [08]:

$$
\sigma_{\theta q}=\frac{\sigma}{\left(1+\frac{2 R}{a}\right) \ln \left(1+\frac{a}{2 R}\right)}=F_{T}, \sigma
$$

with

$$
F_{T}=\left[\left(1+\frac{2 R}{a}\right) \ln \left(1+\frac{a}{2 R}\right)\right]^{-1}
$$

which may be considered as the nominal stress $(\sigma)$ corrected by a factor $F_{T}$.

Based on the same three assumptions Bridgman extended his correction method to flat bars. However, necking for flat samples proved to be much more complicated than for rods with their circular cross section. For thin strips, two types of necking or flow instability must be considered. The first of them is diffuse necking, so called because its extent is much greater than the sample thickness.

\section{RESULTS AND DISCUSSION}

A comparison of experimental results with those of models of G'sell [03] on notched specimens is given in (Figure 7), We recall that the model of G'sell allows to predict plastic instability with the introduction of some criterion of plasticity. 
This analysis contributed to a better understanding of the evolution of the behavior of polymers. It is important to note that the amorphous structure of the material, which necessarily plays a very important role in the process of instability (pulling hook), has been neglected.

Fig. 7 to 10 show the results of the evolution of the true stress corrected by a factor of triaxiality depending on the true strain for four radiuses proposed in this study (R2 to R80), they are compared with the experimental results and theoretical results obtained by modeling of G'sell [03].

We can observe a good correlation of predictions with experimental and modeling results, except the R2 where a larger gap appears in the curing phase between the corrected curve and the theoretical curves.

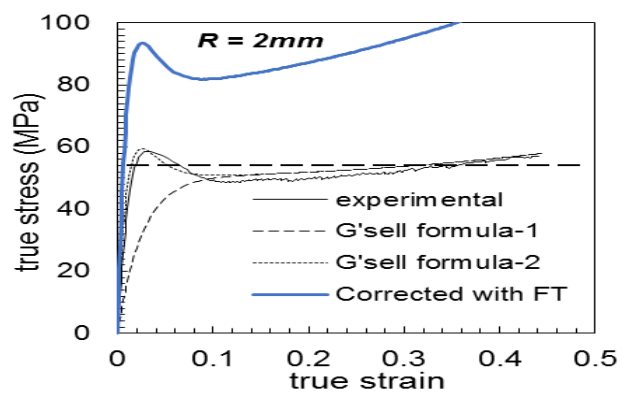

Figure 7. Evolution of the true stress vs. true strain for a radius of curvature $\mathrm{R}=2 \mathrm{~mm}$.

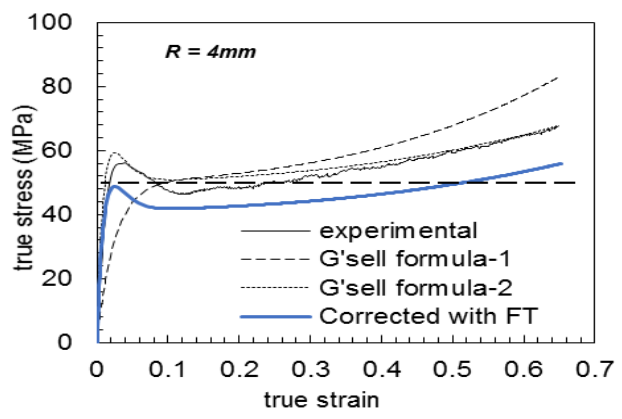

Figure 8. Evolution of the true stress vs. true strain for a radius of curvature $R=4 \mathrm{~mm}$

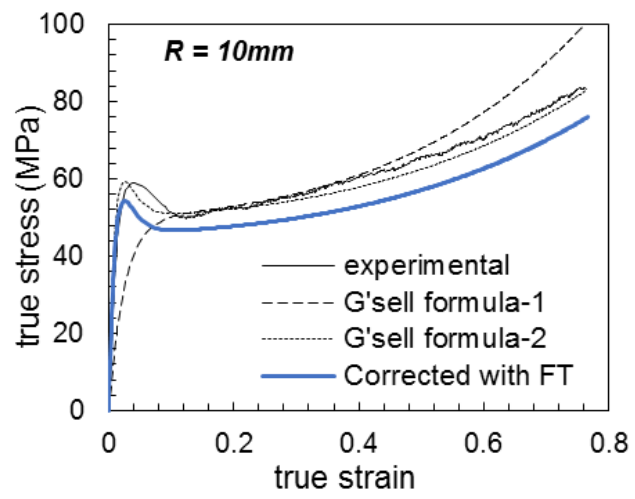

Figure 9. Evolution of the true stress vs. true strain for a radius of curvature $\mathrm{R}=10 \mathrm{~mm}$

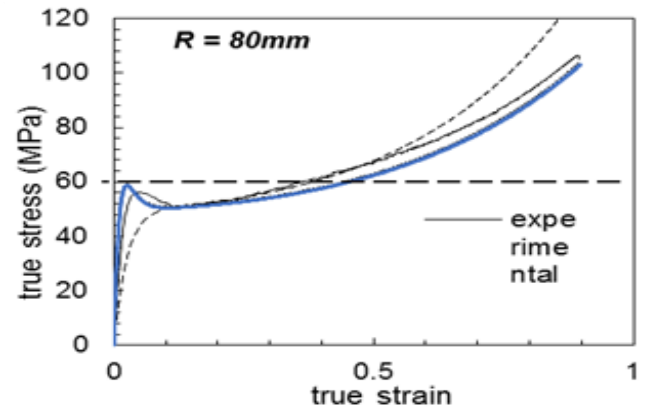

Figure 10. Evolution of the true stress vs. true strain for a radius of curvature $\mathrm{R}=80 \mathrm{~mm}$

\section{CONCLUSION}

The propagation of a constriction along an otherwise uniform section of the sample can be explained in terms of the transverse compressive stresses developed at the current shoulders of the neck. The effect of these triaxle stresses can be quantified in terms of a modified Bridgman correction factor, which is seen to be valid in locations of negative as well as positive curvature. In the presence of variations in cross-section, the gradient in the Bridgman triaxiality factor must be added to the strain localization relationship and plays a role analogous to that of an area or strength defect.

\section{REFERENCES}

[1] Dreistadt C. (2007). Analyse expérimentale et modélisation micromécanique du comportement du polycarbonate soumis aux chargements complexes.

[2] Ebewele R.O. (1996). Polymer-science-andtechnology.

[3] G'sell C. (1988). Instabilités de déformation pendant l'étirage des polymères solides, Revue de Physique Appliquée, Vol. 23, No. 6, pp. 1085-1101.

[4] G'sell C., Aly-Helal N.A., Jonas J.J. (1983). Effect of stress triaxiality on neck propagation during the tensile stretching of solid polymers, Journal of Materials Science, Vol. 18, No. 6, pp.1731-1742.

[5] Hosford W.F. (2005). Mechanical behavior, testing, and manufacturing properties of materials.

[6] Jonas J.J., Holt R.A., et al. (1976). Plastic stability in tension and compression, Acta Metallurgica, Vol. 24, No.10, pp. 911-918.

[7] Ling Y. (1996). Uniaxial true stress-strain after necking, AMP Journal of Technology, Vol. 5, pp. 3748.

[8] Tsuchida N., Inoue T., Enami K. (2011). Estimations of the true stress and true strain until just before fracture by the stepwise tensile test and Bridgman equation for various metals and alloys, Materials Transactions, Vol. 53, No. 1, pp. 133-139.

[9] Lechêne S. (2013). Etude expérimentale et simulation numérique de l'effet de la triaxialité sur le comportement mécanique du PVC, Chapitre-II, pp. 56-93. 